\title{
Urinoma as an initial presentation of rectosigmoid cancer
}

\author{
M.M.Wani ${ }^{1}$, A.Rashid ${ }^{2}$, R.S.Bali ${ }^{3}$, R.A.Bhat ${ }^{4}$ \\ ${ }^{1,4}$ Government Medical College, Srinagar \\ ${ }^{2,3}$ Maulana Azad Medical College, New Delhi, India
}

Key words: Urinoma; ureter; adenocarcinoma; signet ring cell cancer; rectosigmoid.

\begin{abstract}
Colorectal cancer is the most common primary malignancy of the gastrointestinal tract and may present with a myriad of symptoms. We describe a case of a 36 year old man who presented with a urinoma on the right side secondary to ureteric obstruction at the pelvic brim. The urinoma did not resolve by conservative measures and a laparotomy was performed this revealed an unsuspected recto-sigmoid adenocarcinoma compressing the right lower ureter producing the urinoma. An anterior resection, along-with en bloc resection of the involved ureter and a ureteroureterostomy, was performed. This case illustrates that a rectal cancer may masquerade as a urinoma and surgeons should keep in mind pelvic causes, particularly a mitotic lesion of the rectosigmoid, as a differential diagnosis in such cases so that proper management ensues.
\end{abstract}

\section{Introduction}

The colon and the rectum are the most common sites of primary gastrointestinal malignancy [1]. There are various presentations of rectosigmoid malignancies, include bleeding, constipation, tenesmus, change in the caliber of stools and altered bowel habits. Cases may also present as a mass in the left iliac fossa, large bowel obstruction or as feacal peritonitis following perforation. These presentations reflect various stages

Correspondence: A.Rashid

E-mail: arsh002@gmail.com of a locally expanding tumour [1]. We report a case of recto-sigmoid (signet ring cell) adenocarcinoma found unexpectedly in a patient who presented to us with urinary symptoms and was diagnosed to have a urinoma on the right side. To the best of our knowledge this is only the second case report in the literature.

\section{Case presentation}

A 36 year old male presented to the emergency department with a three week history of pain in the right lumbar region, radiating to the groin. Clinical examination was unremarkable except for fullness in the right flank. Baseline blood investigations were normal. Abdominal ultrasonography was done and revealed a fluid collection in relation to the right kidney. An ultrasound guided diagnostic tap revealed an aspirate suggestive of urine which was confirmed on biochemistry. An urgent intravenous urogram revealed hydronephrosis on the right side with a column of contrast in the proximal part of the right ureter. Extravasation of contrast was noted around the kidney extending up to the lower one third of the ureter (Figure 1). Cystoscopy with right retrograde pyelography revealed an extrinsic compression without intraluminal pathology. A contrast enhanced computed tomogram (CECT) of the abdomen confirmed the presence of a homogenous fluid collection, with contrast enhancement, in relation to the right kidney extending inferiorly (Figure 2). A preliminary diagnosis of a right sided urinoma secondary to lower ureteric obstruction was made. 
The urinoma was drained percutaneously under ultrasound guidance. An attempt to place a cystoscopic guided ureteric stent on the right side was unsuccessful. After two weeks, the patient re-presented with the same symptoms. The urinoma had re-accumulated and the patient underwent right lower ureteric exploration which, revealed a recto-sigmoid growth causing extrinsic compression of the lower right ureter. A formal laparotomy was performed. There was no clinical evidence of metastatic disease in the abdominal cavity. A high anterior resection of the rectum was performed with en-bloc ureteric resection and reconstruction over a stent. Pathological examination revealed a poorly differentiated adenocarcinoma of the recto-sigmoid with signet ring cell pattern. The patient recovered uneventfully and was discharged on the seventh postoperative day with a plan to receive adjuvant chemotherapy This patient completed six cycles of chemotherapy successfully, and is well thirteen months later.

\section{Discussion}

A urinoma is an encapsulated extra- pelvicalyceal collection of urine that forms due to leakage through a tear in the collecting system or the proximal ureter when ureteric obstruction is present. The extravasated urine causes lipolysis and inflammation of peri-renal fat with formation of a fibrous capsule around the collected urine [2]. Most commonly, renal urine leaks result from blunt or penetrating renal trauma- the so called nonobstructive urinomas [3].

A colorectal malignancy presenting initially as an urinoma has been reported only once in the English literature and on two occasions in non-English literature [4]Sayeed et al reported a case of urinoma due to a rectal cancer but said the patient was already suffering from Hodgkin's disease and metastatic disease [4]. Guzman-Fernandez et al reported a sigmoid cancer locally infiltrating the ureter and causing a urinoma but the primary tumour was sought only postoperatively after the histopathology revealed involvement of the ureter; despite the patient giving a history of constipation [5]. Sakaguchi et al reported the occurrence of a urinoma due to retroperitoneal lymph node metastases secondary to adenocarcinoma of ascending colon [6]. Signet ring cell cancers (SRCC) are aggressive and tend to involve the full thickness of the bowel early [7]. Therefore it has a high propencity to infiltrate adjacent structures. Our patient did not have clinical features suggestive of colorectal malignancy at presentation, which is a feature of SRCC [8] . Histopathology failed to show infiltration into the ureter and there were no distant metastases on laparotomy or subsequent metastatic work-up.

Abdominal ultrasound can identify a urinoma as an anechoic localized collection [2]. A CECT scan reveals a homogenous hypodense well encapsulated lesion in relation to kidneys with increase in attenuation values upon intravenous contrast injection [9]. Renal scintigraphy with $99 \mathrm{mTc}$-DTPA may delineate the site and estimate the leakage rate [10]. Treatment of urinoma is dependent on its cause. Most non-obstructive urinomas resolve with aspiration, stenting or percutaneous nephrostomy [9]. However, in obstructive causes, surgery may be indicated [2]. In thispatient there was an obstruction to the distal ureter through which a ureteric stent could not be negotiated, so a decision for exploration was taken. In rare cases a nephrectomy may be warranted to treat an urinoma.

\section{Conclusion}

Despite previous reports, such a presentation of rectal adenocarcinoma is not recognized widely, and may cause delays in diagnosis and management. Hence, we are of the opinion that whenever a patient with urinoma is being evaluated, pelvic causes, particularly a mitotic lesion of colon should be considered in the differential diagnosis. 


\section{References}

1. Dalal KM, Bleda R. Cancer of the Rectum. In: Zinner MJ, Ashley SW, eds. Maingot's Abdominal Operations, 11th ed. New York: McGraw Hill; 2007: 693-726.

2. Rizvi S, Ibne A, Siddiqui MA, Syed M. A case report: Urinoma as initial presenting sign of bladder malignancy. Indian J Cancer 2011;48:516-7.

3. Ito S, Ikeda M, Asanuma $H$, Shishido S, Nakai H, Honda M. A giant urinoma in a neonate without obstructive uropathy. Pediatr Nephrol 2000;14:831-2.

4. Sayeed R, Nyamekye I, Kinder R. Unsuspected rectal adenocarcinoma causing urinoma. Int J Urol 1997;4:99100.

5. Guzman-Fernandez A, Cortadella-Angel R, PerezHerms S, Alberola-Ferranti M, Castellanos-Acosta R, Alameda-Quitlet F, et al. Urinary tract involvement as the first clinical manifestation of adenocarcinoma of the colon [In Spanish]. Arch Esp Urol 1993;46(2):149-152.
6. Sakaguchi H, Seguchi T, Kajikawa H, Nishioka T, Takada M. A case of spontaneous peripelvic extravasation associated with ureteral stenosis caused by retroperitoneal lymph node metastasis of ascending colon cancer [In Japanese]. Hinyokika kiyo 1987;33:1100-1104.

7. T. Anthony, R. George, M. Rodriguez-Bigas, N. J. Petrelli . Primary signet-ring cell carcinoma of the colon and rectum.. Ann Surg Oncol. 1996 July; 3(4): 344-348.

8. Kohli, R., Singh, S., Gupta, A. K., \& Matreja, P. S. A Case Report of Signet-Ring Cell Carcinoma Colon. Journal of Postgraduate Medicine, Education and Research. 2013;47(3): 153-155

9. Titton RL, Gervais DA, Hahn PF, Harisinghani MG, Arellano RS, Mueller PR. Urine leaks and urinomas: diagnosis and image guided intervention. Radiographics 2003;23(5):1133-1147.

10. Kumar R, Gupta R, Khullar S, Sharma S. The management of urinoma: the role of renal scintigraphy. BJU International 2000;85(9):1154.

\section{Key learning points}

- The left ureter is more likely to be infiltrated by a rectosigmoid tumour compared with the right ureter.

- Quality contrast enhanced computerized tomography should enable identification of extra-ureteric aetiology of ureteric compression. 